\title{
Interfacial Tension of the Lipid Membrane Formed from Phosphatidylcholine-Decanoic Acid and Phosphatidylcholine-Decylamine Systems
}

\author{
Aneta D. Petelska $\cdot$ Zbigniew A. Figaszewski
}

Received: 1 November 2010/Accepted: 22 April 2011/Published online: 11 May 2011

(C) The Author(s) 2011. This article is published with open access at Springerlink.com

\begin{abstract}
Interfacial tension has been determined for phosphatidylcholine (PC)-decanoic acid (DA) and PC-decylamine (DE) membranes. PC (lecithin), DA and $\mathrm{DE}$ were used in the experiments; the interfacial tension values of the pure components are $1.62 \times 10^{-3}$, $-2.38 \times 10^{-2}$ and $-3.88 \times 10^{-2} \mathrm{~N} / \mathrm{m}$ (hypothetical values for DA and DE), respectively. The 1:1 complexes were formed during formation of PC-DA and PC-DE membranes. The following parameters describing the complexes were determined: the surface concentrations of the lipid membranes formed from these complexes, $A_{3}^{-1}$; the interfacial tensions of such membranes, $\gamma_{3}$; and the stability constants of these complexes, $K$.
\end{abstract}

Keywords Interfacial tension - Phosphatidylcholine . Decanoic acid · Decylamine $\cdot$ Complex 1:1

\section{Introduction}

Biological membranes generally contain a mixture of various types of amphiphilic molecules, such as phospholipids, glycolipids, fatty acids, glycerides and amines. These components vary in chain length, saturation and type of chain linkage. Although present at levels of only a few percent, fatty acids and amines are important constituents

\footnotetext{
A. D. Petelska $(\bowtie) \cdot$ Z. A. Figaszewski

Institute of Chemistry, University of Bialystok, Al. J. Pilsudskiego 11/4, 15-443 Bialystok, Poland

e-mail: aneta@uwb.edu.pl

\section{Z. A. Figaszewski}

Laboratory of Electrochemical Power Sources,

Faculty of Chemistry, University of Warsaw, Pasteur St. 1,

02-093 Warsaw, Poland
}

of biomembranes (Kumar et al. 2005). They can affect any physiological function, such as enzyme activity (Gennis 2010), particularly the activation of lipid-metabolizing enzymes, and calcium transport (Felix 2006).

Since fatty acids belong to the lipids that form biological membranes and at the same time also possess a quite simple structure, they are frequently used by researchers for modeling. It is clear that there is a certain equilibrium between the lipid-forming bilayer and lipid molecules and substances present in electrolyte solution. The interactions between membrane lipids are studied by several techniques; however, quantitative descriptions of the systems are lacking. Formation of artificial membranes with built-in study components allows us to research in simpler systems than complicated biological membranes.

Many investigations have been reported on the effect of fatty acids on physical and chemical properties of model membranes, particularly on the phase behavior of hydrated phospholipid bilayers, using various techniques, such as differential thermal analysis (Ulkowski et al. 2005), differential scanning calorimetry (Ohta and Hatta 2002; Matricarde Falleiro et al. 2010; Inoue et al. 2001), fluorescence spectroscopy (Borst et al. 2000), X-ray diffraction (Kumar et al. 2005), magnetic resonance (Heimburg et al. 1990; Rama Krishna and Marsh 1990) and Fourier transform infrared spectroscopy (Inoue et al. 2001).

In this work, the interfacial tension of phosphatidylcholine (PC)-decanoic acid (DA) and PC-decylamine (DE) membranes was determined within the entire composition range where bilayer formation was possible.

The aim of these investigations was to study the mixed PC-DA and PC-DE bilayer, characterize the molecular interaction between phospholipids and fatty acids and between phospholipids and amine and compare the properties of these systems: stability constants of the formed 
complexes and surface areas occupied by pure membrane components.

\section{Theory}

In cases where the membrane components do not form chemical compounds, their interaction can be described by the following set of equations (Petelska and Figaszewski 1998; Petelska et al. 2006a):

$$
\begin{aligned}
& \gamma_{1} m_{1} A_{1}+\gamma_{2} m_{2} A_{2}=\gamma \\
& \frac{m_{1}}{m_{1}+m_{2}}=x_{1} \\
& x_{1}+x_{2}=1
\end{aligned}
$$

where $A_{1}^{-1}, A_{2}^{-1}\left(\mathrm{~mol} \mathrm{~m}^{-2}\right)$ are the surface concentrations of components 1 and $2 ; m_{1}, m_{2}\left(\mathrm{~mol} \mathrm{~m}{ }^{-2}\right)$ are the quantities of components 1 and 2 per unit area of the membrane; $\gamma_{1}, \gamma_{2}\left(\mathrm{~N} \mathrm{~m}^{-1}\right)$ are the interfacial tensions of membranes assembled from pure components 1 and $2 ; \gamma\left(\mathrm{N} \mathrm{m}^{-1}\right)$ is the measured interfacial tension of the membrane; and $x_{1}, x_{2}$ are the solution mole fractions of components 1 and 2 .

Elimination of $m_{1}$ and $m_{2}$ yields the following linear equation:

$\left(\gamma-\gamma_{1}\right) x_{1}=\frac{A_{2}}{A_{1}}\left(\gamma_{2}-\gamma\right) x_{2}$

Membranes may also be assembled from two components capable of forming a complex. The stoichiometry of the complex may vary, but because the first stability constant in these complexes is usually the largest (Inczedy 1976; Beck and Nagypal 1990), we assumed that the complexes are primarily of 1:1 stoichiometry.

In cases where the membrane components form a 1:1 complex, interactions in the membrane may be described by a previously published set of equations (Petelska et al. 2006a, b).

The equilibrium between the individual components and the complex is represented by

$$
\mathrm{A}(\text { Component } 1)+\mathrm{B}(\text { Component } 2) \Leftrightarrow \mathrm{AB}(\text { Complex })
$$

and the basic equation describing the interaction between components 1 and 2 can be written as follows (Petelska et al. 2006a, b):

$$
\begin{aligned}
& {\left[\left(\gamma-\gamma_{1}\right) B_{2} x_{1}+\left(\gamma-\gamma_{2}\right) B_{1} x_{2}\right]\left[\left(\gamma_{3}-\gamma_{1}\right) B_{2} x_{1}\right.} \\
& \left.\quad+\left(\gamma_{3}-\gamma_{2}\right) B_{1} x_{2}+\left(\gamma_{1}-\gamma_{2}\right)\left(x_{1}-x_{2}\right)\right] \\
& \quad=K A_{3}^{-1} B_{1} B_{2}\left[\left(\gamma-\gamma_{1}\right)\left(x_{2}-x_{1}\right)+\left(\gamma_{3}-\gamma\right) B_{1} x_{2}\right] \\
& \quad \times\left[\left(\gamma-\gamma_{2}\right)\left(x_{1}-x_{2}\right)+\left(\gamma_{3}-\gamma\right) B_{2} x_{1}\right]
\end{aligned}
$$

where $B_{1}=A_{3} / A_{1}$ and $B_{2}=A_{3} / A_{2}$.

Equation 3 may be simplified by taking into account the high stability constant of the complex. Applying this simplification results in linear behavior for small $\left(x_{2}<x_{1}\right)$ and large $\left(x_{2}>x_{1}\right) x_{2}$ values. When calculating the stability constant for the complex, Eq. 3 can be simplified to $x_{1}=x_{2}$ (Petelska et al. 2006a, b).

The parameters describing the complex may be used to calculate theoretical points using the equation presented below (agreement between the theoretical and experimental values implies that the system is well described by the above equations):

$$
\begin{aligned}
K A_{1}^{-1} & A_{2}^{-1}\left(a_{1}+a_{2}\right)\left(a_{3}-a_{1}\right) \gamma^{2} \\
+ & {\left[K A_{1}^{-1} A_{2}^{-1}\left(\gamma_{1} a_{1}-\gamma_{3} a_{3}\right)\left(a_{1}+a_{2}\right)\right.} \\
& -K A_{1}^{-1} A_{2}^{-1}\left(\gamma_{2} a_{1}+\gamma_{3} a_{2}\right)\left(a_{3}-a_{1}\right) \\
& \left.+a_{4} A_{3}^{-1}\left(a_{3}+a_{2}\right)\right] \gamma \\
& +K A_{1}^{-1} A_{2}^{-1} a_{3} \gamma_{3}\left(\gamma_{3} a_{2}+\gamma_{2} a_{1}\right) \\
& -K A_{1}^{-1} A_{2}^{-1} a_{1} \gamma_{1}\left(a_{1} \gamma_{2}+a_{2} \gamma_{3}\right) \\
& -a_{4} A_{3}^{-1}\left(\gamma_{2} a_{3}+\gamma_{1} a_{2}\right)=0 \\
a_{1}= & A_{3}^{-1}\left(x_{2}-x_{1}\right) \\
a_{2}= & A_{2}^{-1} x_{1} \\
a_{3}= & A_{1}^{-1} x_{2} \\
a_{4}= & {\left[A_{3}^{-1}\left(\gamma_{1}-\gamma_{2}\right)\left(x_{2}-x_{1}\right)\right.} \\
& \left.+\left(\gamma_{1}-\gamma_{3}\right) x_{1} A_{2}^{-1}+\left(\gamma_{2}-\gamma_{3}\right) x_{2} A_{1}^{-1}\right]
\end{aligned}
$$

For systems containing two lipid components, 1:1 complex formation was assumed to be the explanation for deviation from the additivity rule. Model curves were constructed using calculated parameters such as equilibrium constants, molecular areas of the complexes and the interfacial tension of molecules and complexes. The accuracy of the models was verified by comparison to experimental results.

\section{Experimental Procedure}

Measuring Apparatus and Measuring Procedures

The interfacial tension method is based on Young and Laplace's equation. The tension in a lipid bilayer sample is determined by measuring the radius of curvature of the convex surface formed when a pressure difference is applied across the bilayer (Adamson 1960).

The apparatus and measurement method were described previously (Petelska and Figaszewski 1998; Petelska et al. 2006a, b). The measurement system consists of two glass chambers separated by a mount holding a 1.5 -mm-diameter circular Teflon element axially pierced by a small orifice.

Membranes were formed by the Mueller-Rudin method (Tien and Ottova-Leitmannova 2003) on the flat end of the Teflon element. Both chambers were filled with an electrolyte solution. The membrane-forming solution was 
introduced to the flat wall of the Teflon element using a micropipette, and pressure was applied to the left chamber using a manometer.

The convexity of the lipid membrane cap was measured to $0.05 \mathrm{~mm}$ precision. The radius of curvature was determined using this value and the diameter of the Teflon element, corresponding to diameter of the lipid cap.

\section{Reagents}

The following reagents were used for preparation of the membrane-forming solution:

1. 3-sn-phosphatidylcholine (99\%; Fluka, Buchs, Switzerland) isolated from hen egg yolk

2. Decanoic acid $(98 \%$, Fluka)

3. Decylamine $(99.5 \%$, Fluka)

The as-received PC and DA were purified by dissolving in chloroform and evaporating the solvent under argon. The stock membrane-forming solutions consisted of $20 \mathrm{mg} \mathrm{cm}^{-3}$ of the desired lipids (PC, DA or DE) in 20:1 $n$-decane:butanol. The solution containing the membrane components was not saturated and could therefore contain the components in any proportion. During membrane formation, the solvent was removed, leaving a membrane composed of lipids in the same ratio as the stock solution.

The electrolyte solution contained $0.1 \mathrm{M}$ potassium chloride and was prepared using triple-distilled water and $\mathrm{KCl}$, produced by POCh (Gliwice, Poland). The $\mathrm{KCl}$ was calcined to remove any organic impurities.

All solvents were chromatographic standard grade. The $n$-decane was purchased from Merck (Darmstadt, Germany), and the chloroform and butanol were obtained from Aldrich (Milwaukee, WI).

All experiments were carried out at $293 \pm 2 \mathrm{~K}$.

\section{Results and Discussion}

The effect of the presence of DA or DE on interfacial tension of the membranes formed from PC was studied. The dependence of interfacial tension on the lipid membrane as a function of composition was studied at room temperature $(293 \pm 2 \mathrm{~K})$ in the entire feasible concentration range. The interfacial tension values reported here refer to the two sides of the bilayer membrane surface area unit.

Figure 1 contains a graph of $\left(\gamma-\gamma_{1}\right) x_{1}$ vs. $\left(\gamma_{2}-\gamma\right) x_{2}$ for the four systems PC-DA (Fig. 1a) and PC-DE (Fig. 1b). According to Eq. 2, when the membrane components do not interact, these functions should yield straight lines. This is clearly not the case, which suggests that a complex or other structure exists in PC-DA and
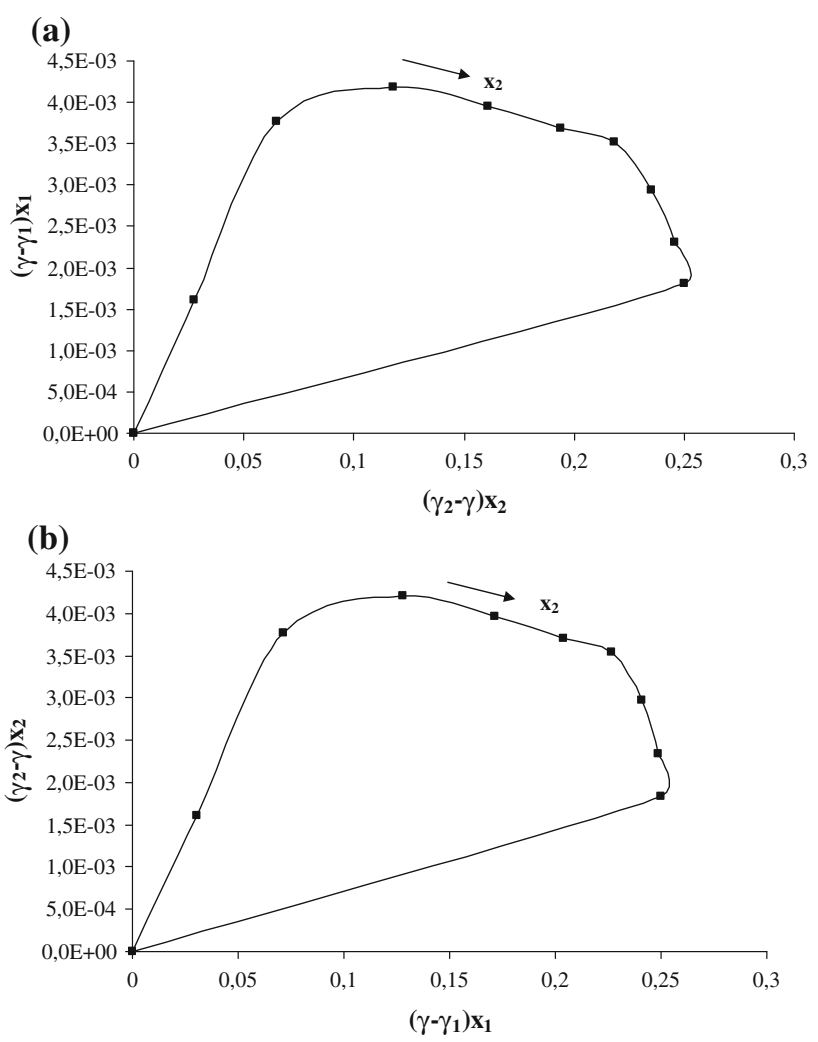

Fig. 1 Graph of Eq. 2 for PC-DA (a) and PC-DE (b), where $x_{2}$ is the mole fraction of component 2 (DA and DE, respectively)

PC-DE bilayers. Because the use of Eq. 3 presupposes the existence of 1:1 complexes, our initial assumption was that the complexes formed were 1:1. The interfacial tension of the lipid membrane was studied over a wide range of lipid compositions.

The dependence of interfacial tension of lipid membrane as a function of membrane composition for PC-DA and PC-DE systems was studied over a possible concentration range. The results are depicted in Fig. 2a, b. The dependence of interfacial tension on the lipid membranes formed from the PC-DA and PC-DE systems was executed in the function of the composition to $41 \%$ of the DA contents (to $40 \%$ of the DE) because only to such contents of component 2 (DA, DE) with lecithin was a bilayer membrane formed.

The interfacial tension value of pure lecithin membrane (component 1), $\gamma_{1}$, was measured directly and presented earlier (Petelska and Figaszewski 1998), which is equal to $1.62 \times 10^{-3} \mathrm{~N} \mathrm{~m}^{-1}$. There are no accurate data on interfacial tension values for the pure component 2 (DA or DE) because these components do not create a bilayer membrane. However, in order to characterize the course of the experimental curves, the $\gamma_{2}$ value for the pure components is necessary, which will be used for calculation. In this case, the interfacial tension hypothetical values for membranes built from DA and DE were determined by adjusting the 
(a)
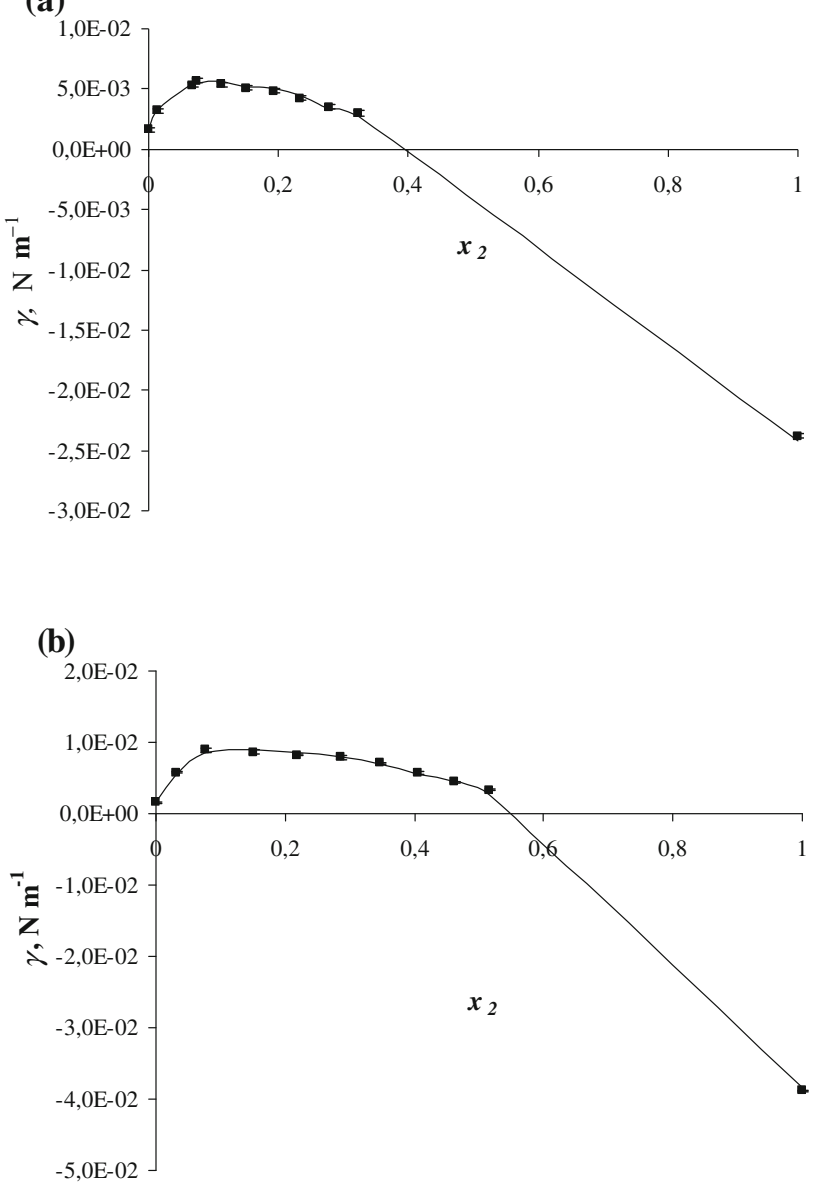

Fig. 2 The interfacial tension, $\gamma$, of the PC-DA (a) and PC-DE (b) membranes as a molar fraction of $\mathrm{DA}$ or $\mathrm{DE}, x_{2}$ (experimental values are marked by points and theoretical ones by curves)

experimental curve with the polynomial of the other mark extrapolating the $x_{2}=1$ value, which is presented in Fig. 3 . The interfacial tension values obtained in this way for pure DA and DE are equal to $-2.38 \times 10^{-2}$ and $-3.88 \times$ $10^{-2} \mathrm{~N} \mathrm{~m}^{-1}$. Negative values of interfacial tension for membrane built from pure DA and DE point to the fact that it is not possible to create bilayer membranes from pure DA or DE. The thermodynamic potential for this bilayer would have a negative value; i.e., the bilayer is not forming.

The other constants- $B_{1}, B_{2}, \gamma_{3}$-were determined by assuming that the values of the stability constant of the PC-DA and PC-DE complexes were sufficient to be simplified, i.e., Eq. 1 to Eqs. 2 and 3. Knowing the $B_{1}, B_{2}$ constants, which were determined from Eqs. 2 and 3, it was possible to calculate the interfacial tension values of the PC-DA and PC-DE complexes, $\gamma_{3}$. The mean values are equal to $7.25 \times 10^{-3} \mathrm{~N} \mathrm{~m}^{-1}$ for PC-DA and $1.52 \times 10^{-2}$ $\mathrm{N} \mathrm{m}^{-1}$ for PC-DE.

Determining the interfacial tension value as a function of the composition made it possible to determine the surface concentrations of the membranes composed of pure
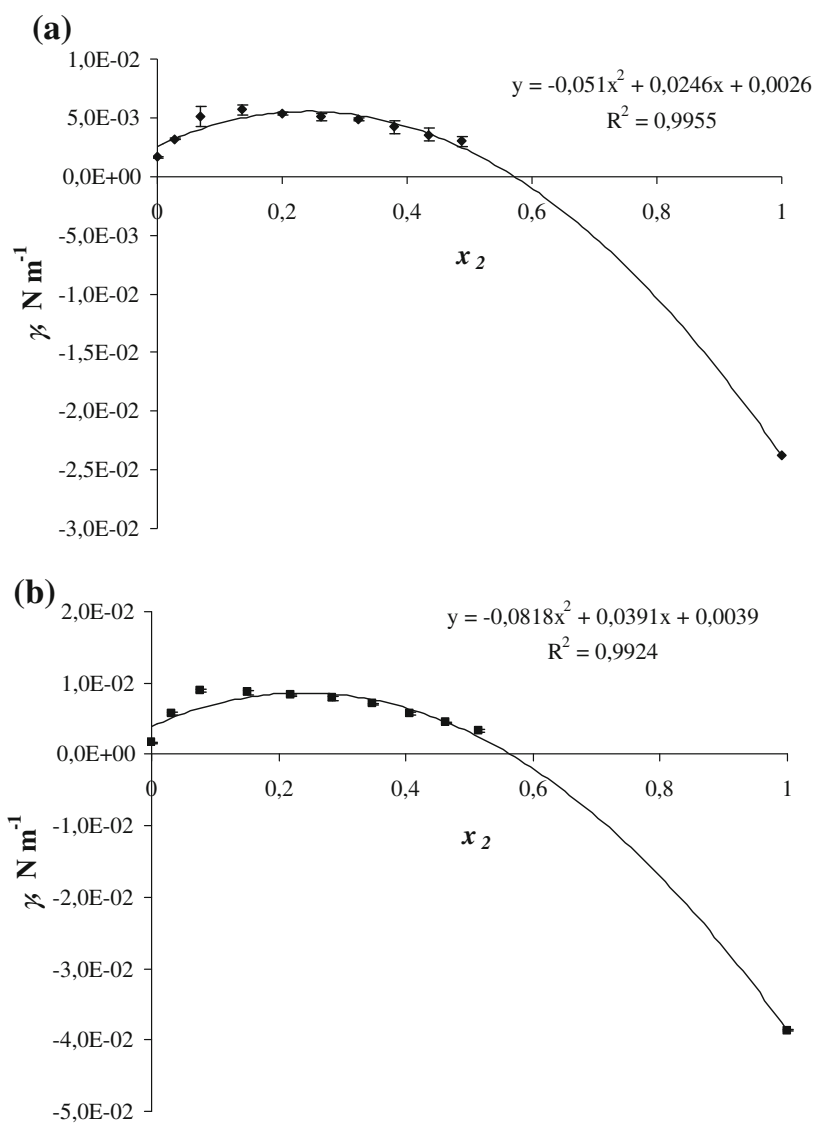

Fig. 3 Plot illustrating the hypothetical interfacial tension values for DA (a) and DE (b) membrane calculation

components. At least one of them is necessary for determination of the $A_{3}^{-1}$ value. The surface area occupied by a lecithin molecule equal to $85 \AA^{2}$ was determined in previous work (Petelska and Figaszewski 2000). The surface areas occupied by DA and DE are equal to 19 and $22 \AA^{2}$, respectively (Petelska and Figaszewski 2011). As mentioned earlier, the fatty acid forms a dimer (Brzozowska and Figaszewski 2003; Zhao and Olesik 2001); therefore, the surface area occupied by DA is equal to $38 \AA^{2}$.

Knowing the $A_{1}^{-1}$ and $A_{2}^{-1}$ as well as $B_{1}$ and $B_{2}$ values, the surface concentration of the membrane composed of the lecithin-DA and lecithin-DE complexes could be determined. The resulting surface concentration values, $A_{3}^{-1}$, for the PC-DA and PC-DE complexes were equal to $1.15 \times 10^{-6}$ and $2.03 \times 10^{-6} \mathrm{~mol} \mathrm{~m}^{-2}$, respectively. This made it possible to determine the area occupied by one lecithin-DA and one lecithin-DE complex, which were 144 and $82 \AA^{2}$, respectively. The value of area occupied by one lecithin-DA complex molecule is larger than the amount of the surface area occupied by each component of the complex. It is probably connected with the arrangement of lecithin molecules in such a complex and with the structural construction of such a complex. Previously 
(Petelska and Figaszewski 2003), we suggested the arrangement of the lecithin molecules in a bilayer membrane at $\mathrm{pH}>5$. In these media, one particle from the lecithin molecule in the bilayer (orientated in this way) has two straightened chains; however, the next molecule of lecithin has one straightened and another chain fastened to the membrane surface. An association of ions occurs in such conditions with $\mathrm{OH}^{-}$from the electrolyte solution. How these ions were characterized was previously reported (Petelska and Figaszewski 2003): These ions are strongly solvated, and they produce a separation of lecithin particles in the bilayer, which increases the surface occupied by the single molecule of lecithin.

The difference between surface area values occupied by one molecule of PC-DA and PC-DE complexes to appear from the fact than as the chain length increases, van der Waals interactions between the chain of the adjacent molecule increase, bringing these molecules closer to each other. When this happens, the carboxylic acid groups of the fatty acids are also packed closer, shielding the hydrogen atom between the two oxygen atoms (Kanicky et al. 2000; Kanicky and Shah 2002).

The only values to be determined were the stability constants of the PC-fatty acid and PC-amine complexes. It could be determined from Eq. 4 when $x_{1}=x_{2}=0.5$; these parameters amount to $4.61 \times 10^{6}$ and $2.38 \times 10^{5} \mathrm{~m}^{2}$ $\mathrm{mol}^{-1}$, respectively. During the course of our investigations, we assumed the formation of PC-DE and PC-DE complexes. These complexes arise by producing a connection between the $-\stackrel{(+)}{\mathrm{N}}\left(\mathrm{CH}_{3}\right)_{3}$ group from the molecule of lecithin and $-\mathrm{COO}^{(-)}$groups from the dimer of DA, in the case of the complex $\mathrm{PC}-\mathrm{DA}$, and between the $-\mathrm{PO}^{(-)}$group from lecithin and the $-\stackrel{(+)}{\mathrm{N}} \mathrm{H}_{3}$ group from $\mathrm{DE}$. The dissociation constants of the $-\stackrel{(+)}{\mathrm{N}}\left(\mathrm{CH}_{3}\right)_{3}$ group from $\mathrm{PC}$ and the $-\mathrm{COO}^{(-)}$groups from the dimer of DA are equal $10^{-5.7}$ (Petelska and Figaszewski 2000) and about $10^{-5}$ (Chemistry Tables 2004), respectively. It should be noted that the dissociation constants of the $-\mathrm{PO}^{(-)}$group from $\mathrm{PC}$ and the $-\stackrel{(+)}{\mathrm{N}_{3}}$ group from DE equal $10^{-2.6}$ (Petelska and Figaszewski 2000) and about $10^{-10}$ (Chemistry Tables 2004), respectively. Therefore, the connection between PC and DA will be stronger, and we can expect that the stability constant of PC-DA will be higher than the stability constant of PC-DE.

The parameters describing these complexes determined from Eqs. 1 and 4 were in agreement with Eq. 1; i.e., data (solid lines) with the experimental data (points) in Fig. 1 using equation 5. As it is a square equation, this equation can yield two solutions. The values yielding a better agreement of the experimental points with equations describing complex formation between membrane lipid components were chosen.

The experimental values in Fig. 1 are marked by points and the theoretical ones obtained from equation 5 , by lines. It can be seen from this figure that there is a good agreement between experimental and theoretical points, which verifies the assumption of formation of 1:1 PC-DA and PC-DE complexes in the lipid membrane. Good agreement of the experimental and theoretical points verifies the assumption of the correct choice of the $\gamma_{2}$ values for components of the membrane.

Table 1 lists several physicochemical parameters for membranes containing PC-DA and PC-DE complexes.

\section{Conclusion}

The following conclusions can be drawn from the parameters describing the complexes studied:

1. The stability constant of the PC-DA complex is $4.61 \times 10^{6} \mathrm{~m}^{2} \mathrm{~mol}^{-1}$, whereas the stability constant of the PC-DE complex is $2.38 \times 10^{5} \mathrm{~m}^{2} \mathrm{~mol}^{-1}$. High values confirm the legitimacy of simplifying Eq. 1 . The values of the stability constants of the lipid-DA and lipid-DE complexes are reported for the first time. It can be observed that the stability constants of the fatty acid-containing complex are higher. Thus, the PC-DA complex is more stable than the PC-DE complex.

2. The experimental area occupied by one PC-DA complex is $144 \AA^{2}$, whereas the area occupied by the PC-DE complex is $82 \AA^{2}$.

3. Good agreement of the experimental and theoretical points verifies the assumption of formation of a $1: 1$ complex in the lipid membrane. A lack of variance between points indicates that complexes at different stoichiometries are not possible in the PC-DA or PCDE membrane.

Table 1 Selected physicochemical parameters for two complexes: phosphatidylcholine-decanoic acid (PC-DA) and phosphatidylcholinedecylamine (PC-DE)

\begin{tabular}{llll}
\hline Examined system & $\begin{array}{l}\text { Surface area occupied by one } \\
\text { molecule of complex }\left(\AA^{2} \text { molecule }{ }^{-1}\right)\end{array}$ & $\begin{array}{l}\text { Stability constant of examined } \\
\text { complex }\left(\mathrm{m}^{2} \mathrm{~mol}^{-1}\right)\end{array}$ & $\begin{array}{l}\text { Complex formation energy } \\
(\mathrm{Gibbs} \text { free energy) } \\
(\mathrm{kJ} \text { mol }\end{array}$ \\
\hline PC-DA & $144 \pm 1.44$ & $4.61 \times 10^{6}$ & $-40.01 \pm 1.20$ \\
PC-DE & $82 \pm 0.82$ & $2.38 \times 10^{5}$ & $-32.45 \pm 0.97$ \\
\hline
\end{tabular}


Open Access This article is distributed under the terms of the Creative Commons Attribution Noncommercial License which permits any noncommercial use, distribution, and reproduction in any medium, provided the original author(s) and source are credited.

\section{References}

Adamson AW (1960) Physical chemistry of surfaces. Interscience Publishers, New York, pp 4-9

Beck MT, Nagypal I (1990) Complex equilibria: stability constants. Ellis Horwood series in inorganic chemistry. Ellis Horwood, Chichester, UK

Borst JW, Visser NV, Kouptsova O, Visser AJWG (2000) Oxidation of unsaturated phospholipids in membrane bilayer mixtures is accompanied by membrane fluidity changes. Biochim Biophys Acta 1487:61-73

Brzozowska I, Figaszewski ZA (2003) Palmitic acid dimer formation in the monolayers at the air/aqueous solution interface. Colloids Surf B 30:187-192

Chemistry Tables (2004) Adamantan Publishers, Warsaw

Felix R (2006) Calcium channelopathies. Neuromolecular Med 8: 307-318

Gennis RB (2010) Biomembranes: molecular structure and function. Springer-Verlag, New York

Heimburg T, Ryba NJP, Würz U, Marsh D (1990) Phase transition from a gel to a fluid phase of cubic symmetry in dimyristoylphosphatidylcholine/myristic acid $(1: 2, \mathrm{~mol} / \mathrm{mol})$ bilayers. Biochim Biophys Acta 1025:77-81

Inczedy J (1976) Analytical applications of complex equilibria. Akademiai Kiado, Budapest

Inoue T, Yanagihara S, Misono Y, Suzuki M (2001) Effect of fatty acids on phase behavior of hydrated dipalmitoylphosphatidylcholine bilayer: saturated versus unsaturated fatty acids. Chem Phys Lipids 109:117-133

Kanicky JR, Shah DO (2002) Effect of degree, type, and position of unsaturation on the $\mathrm{p} K_{a}$ of long-chain fatty acids. J Colloid Inter Sci 256:201-207

Kanicky JR, Poniatowski AFN, Mehta R, Shah DO (2000) Cooperativity among molecules at interfaces in relation to various technological processes: effect of chain length on the $\mathrm{p} K_{\mathrm{a}}$ of fatty acid salt solutions. Langmuir 16:172-177
Kumar V, Fausto N, Abbas A (2005) Robbins \& Cotran pathologic basis of disease. Elsevier Saunders, Philadelphia

Matricarde Falleiro RM, Meirelles AJA, Krähenbühl MA (2010) Experimental determination of the (vapor + liquid) equilibrium data of binary mixtures of fatty acids by differential scanning calorimetry. J Chem Therm 42:70-77

Ohta N, Hatta I (2002) Interaction among molecules in mixtures of ceramide/stearic acid, ceramide/cholesterol and ceramide/stearic acid/cholesterol. Chem Phys Lipids 115:93-105

Petelska AD, Figaszewski ZA (1998) Interfacial tension of the twocomponent bilayer lipid membrane modelling of cell membrane. Bioelectrochem Bioenerg 46:199-204

Petelska AD, Figaszewski ZA (2000) Effect of $\mathrm{pH}$ on the interfacial tension of lipid bilayer membrane. Biophys J 78:812-817

Petelska AD, Figaszewski ZA (2003) Acid-base equilibria at interface separating electrolyte solution and lipid bilayer formed from phosphatidylcholine. Biophys Chem 104:13-19

Petelska AD, Figaszewski ZA (2011) The equilibria of phosphatidylcholine-fatty acid and phosphatidylcholine-amine in monolayers at the air/water interface. Colloids Surf B 82:340-344

Petelska AD, Naumowicz M, Figaszewski ZA (2006a) Physicochemical insights into equilibria in bilayer lipid membranes. In: Tien HT, Ottova A (eds) Advances in planar lipid bilayers and liposomes, vol 3. Elsevier, Amsterdam, pp 125-187

Petelska AD, Naumowicz M, Figaszewski ZA (2006b) The interfacial tension of the lipid membrane formed from lipid-cholesterol and lipid-lipid systems. Cell Biochem Biophys 44:205-211

Rama Krishna YVS, Marsh D (1990) Spin label ESR and ${ }^{31}$ P-NMR studies of the cubic and inverted hexagonal phases of dimyristoylphosphatidylcholine/myristic acid $(1: 2, \mathrm{~mol} / \mathrm{mol})$ mixtures. Biochim Biophys Acta 1024:89-94

Tien HT, Ottova-Leitmannova A (2003) Planar lipid bilayers (BLM's) and their applications advanced in planar lipid bilayers and liposomes. Elsevier, Amsterdam

Ulkowski M, Musialik M, Litwinienko G (2005) Use of differential scanning calorimetry to study lipid oxidation. 1. Oxidative stability of lecithin and linolenic acid. J Agric Food Chem 53: 9073-9077

Zhao J, Olesik SV (2001) Separation of dimer acids using enhancedfluidity liquid chromatography. Anal Chim Acta 449:221-236 